\title{
Salvianolic acid A attenuates vascular remodeling in a pulmonary arterial hypertension rat model
}

\author{
Yu-cai CHEN ${ }^{1}$, Tian-yi YUAN ${ }^{1}$, Hui-fang ZHANG ${ }^{1}$, Dan-shu WANG ${ }^{1}$, Yu YAN ${ }^{1}$, Zi-ran NIU¹, Yi-huang LIN $^{1}$, Lian-hua FANG ${ }^{1, *}$, \\ Guan-hua DU ${ }^{2, *}$ \\ ${ }^{1}$ State Key Laboratory of Bioactive Substances and Functions of Natural Medicines, ${ }^{2}$ Beijing Key Laboratory of Drug Targets \\ Identification and Drug Screening, Institute of Materia Medica, Chinese Academy of Medical Sciences and Peking Union Medical \\ College, Beijing 100050, China
}

\begin{abstract}
Aim: The current therapeutic approaches have a limited effect on the dysregulated pulmonary vascular remodeling, which is characteristic of pulmonary arterial hypertension (PAH). In this study we examined whether salvianolic acid A (SAA) extracted from the traditional Chinese medicine 'Dan Shen' attenuated vascular remodeling in a PAH rat model, and elucidated the underlying mechanisms.

Methods: PAH was induced in rats by injecting a single dose of monocrotaline (MCT $60 \mathrm{mg} / \mathrm{kg}$, sc). The rats were orally treated with either SAA $\left(0.3,1,3 \mathrm{mg} \cdot \mathrm{kg}^{-1} \cdot \mathrm{d}^{-1}\right)$ or a positive control bosentan $\left(30 \mathrm{mg} \cdot \mathrm{kg}^{-1} \cdot \mathrm{d}^{-1}\right)$ for 4 weeks. Echocardiography and hemodynamic measurements were performed on $\mathrm{d} 28$. Then the hearts and lungs were harvested, the organ indices and pulmonary artery wall thickness were calculated, and biochemical and histochemical analysis were conducted. The levels of apoptotic and signaling proteins in the lungs were measured using immunoblotting.

Results: Treatment with SAA or bosentan effectively ameliorated MCT-induced pulmonary artery remodeling, pulmonary hemodynamic abnormalities and the subsequent increases of right ventricular systolic pressure (RVSP). Furthermore, the treatments significantly attenuated MCT-induced hypertrophic damage of myocardium, parenchymal injury and collagen deposition in the lungs. Moreover, the treatments attenuated MCT-induced apoptosis and fibrosis in the lungs. The treatments partially restored MCT-induced reductions of bone morphogenetic protein type II receptor (BMPRII) and phosphorylated Smad1/5 in the lungs.

Conclusion: SAA ameliorates the pulmonary arterial remodeling in MCT-induced PAH rats most likely via activating the BMPRII-Smad pathway and inhibiting apoptosis. Thus, SAA may have therapeutic potential for the patients at high risk of PAH.
\end{abstract}

Keywords: salvianolic acid A; Dan Shen; bosentan; monocrotaline; pulmonary artery hypertension; vascular remolding; apoptosis; BMPRII; Smad

Acta Pharmacologica Sinica (2016) 37: 772-782; doi: 10.1038/aps.2016.22; published online 16 May 2016

\section{Introduction}

Pulmonary arterial hypertension $(\mathrm{PAH})$ is characterized by dysregulated pulmonary vascular remodeling, which leads to an increase in pulmonary vascular resistance, right ventricular hypertrophy, uncompensated right heart failure, and ultimate death $^{[1,2]}$. Despite the current therapeutic approaches for improving symptoms, exercise capacity, and hemodynamic variables, these therapies have a limited effect on vascular remodeling and do not confer a mortality benefit ${ }^{[3,4]}$.

Recent studies have demonstrated that the transforming

\footnotetext{
*To whom correspondence should be addressed.

E-mail fanglh@imm.ac.cn (Lian-hua FANG); dugh@imm.ac.cn (Guan-hua DU)

Received 2015-10-05 Accepted 2016-03-08
}

growth factor- $\beta$ /BMPRII superfamily plays a key role in the pathobiology of $\mathrm{PAH}^{[5,6]}$. Mutations in the BMPRII gene have been identified in $>50 \%$ of familial PAH patients and in $10 \%$ to $25 \%$ of idiopathic PAH patients ${ }^{[7]}$. Bosentan, which is a dual ET-1 receptor antagonist, has been widely used in the chronic treatment of most forms of pulmonary arterial hypertension and as an off-label agent in many other forms of $\mathrm{PAH}^{[8,9]}$. Bosentan also decreases the neuronal apoptosis rate after ischemia/reperfusion injury ${ }^{[10]}$; thus, it plays an important role in the pathological process of $\mathrm{PAH}^{[11,12]}$. Therefore, bosentan is also evaluated as a positive control in the present study.

$S$ miltiorrhiza, commonly called "Dan Shen," is a traditional Chinese medicine. This agent is broadly used as a clinical drug to treat cardiovascular diseases ${ }^{[13]}$. Salvianolic acid A (SAA) $((2 R)-3-(3,4-d i h y d r o x y p h e n y l)-2-[(E)-3-[2-[(E)-2-(3,4-d i h y-$ 
droxyphenyl)ethenyl]-3,4-dihydroxyphenyl]prop-2-enoyl] oxypropanoic acid) is one of the most bioactive compounds in $S$ miltiorrhiza and exhibits many pharmacological activities, including antioxidation, myocardial protection, antithrombosis, antifibrosis, and the prevention of diabetes complications ${ }^{[14]}$. Recent reports have indicated that SAA may prevent cardiac remodeling ${ }^{[15]}$ and inhibits endothelial dysfunction and vascular remodeling in spontaneously hypertensive rats ${ }^{[16]}$. Microvascular remodeling is an important step for end-organ damage. However, whether SAA improves pulmonary artery remodeling induced by $\mathrm{PAH}$ remains unknown.

To elucidate the effects of SAA on microvascular remodeling, we estimated the protective effects of SAA on hemodynamics, vascular remodeling and myocardial injury in a MCTinduced PAH rat model.

\section{Materials and methods} Animals

All animal care and experimental procedures were performed in accordance with institutional animal ethical committee guidelines, which conform to the Guide for the Care and Use of Laboratory Animals published by the United States National Institutes of Health.

Male Sprague-Dawley rats (170-190 g, Certificate № SCXK (Beijing) 2012-001) were provided by Vital River Laboratory Animal Center (Beijing, China). The Institute of Animal Care and Use Committee at Chinese Academy of Medical Sciences approved all animal experimental procedures.

The animals were allowed to acclimatize for $3 \mathrm{~d}$ in facilities, where the environment was maintained at $22 \pm 3^{\circ} \mathrm{C}$ and $45 \% \pm 10 \%$ humidity under a regular 12 -h/12-h light/dark schedule. All animals had free access to water and food.

\section{Reagents}

SAA (CAS\#: 96574-01-5) with greater than 97\% purity by HPLC analysis was provided as a lyophilized powder by the Institute of Materia Medica (Beijing, China). MCT, bosentan and pentobarbital sodium were purchased from Sigma (St Louis, MO, USA). Alanine aminotransferase (ALT), aspartate aminotransferase (AST) and lactate dehydrogenase (LDH) kits were purchased from BioSino Biotechnology \& Science Inc (Beijing, China). Endothelin-1 (ET-1) kits were obtained from CUSABIO (Wuhan, China). The anti-rabbit IgG or anti-mouse IgG secondary antibodies, enhanced chemiluminescence (ECL) and loading buffer were obtained from CWBIO (Beijing, China). The rabbit monoclonal anti-rat BMPRII antibody and the mouse monoclonal anti-rat glyceraldehyde 3-phosphate dehydrogenase (GAPDH) antibody were purchased from Santa Cruz Biotechnology (Texas, USA). The rabbit polyclonal anti-rat p-Smad1/5, Bcl-2, and Bax antibodies were purchased from Cell Signaling Technology (Danvers, USA).

\section{Experimental protocol and drug administration}

One hundred and twenty male rats were randomly divided into the following six groups: control group (Normal control, $n=20$ ), model group (MCT-exposed, $n=20$ ), bosentan group
(MCT+Bosentan $\left.30 \mathrm{mg} \cdot \mathrm{kg}^{-1} \cdot \mathrm{d}^{-1}, n=20\right)$, and SAA $(0.3,1,3$ $\mathrm{mg} \cdot \mathrm{kg}^{-1} \cdot \mathrm{d}^{-1}$ ) groups (MCT+SAA 0.3, 1, $\left.3 \mathrm{mg} \cdot \mathrm{kg}^{-1} \cdot \mathrm{d}^{-1}, n=20\right)^{[17]}$. MCT-induced pulmonary hypertension was developed by a single subcutaneous injection of MCT $(60 \mathrm{mg} / \mathrm{kg})^{[18,19]}$, whereas animals in the control group were simultaneously injected with physiological saline. Salvianolic acid A (0.3, 1 , and $3 \mathrm{mg} \cdot \mathrm{kg}^{-1} \cdot \mathrm{d}^{-1}$, dissolved in saline) or bosentan (30 $\mathrm{mg} \cdot \mathrm{kg}^{-1} \cdot \mathrm{d}^{-1}$, suspended in saline) was orally administered every day for 4 weeks after MCT injection, whereas the control and model groups received the same volume of physiological saline.

\section{Echocardiography}

Echocardiography was performed at the end of the study as described previously ${ }^{[20,21]}$. In brief, rats in each group were anesthetized by an intraperitoneal injection of pentobarbital sodium $(40 \mathrm{mg} / \mathrm{kg}$, ip). The depth of anesthesia was evaluated by pinching the animal's paw with forceps, and no supplemental analgesia was needed. The heating panel was introduced to avoid hypothermia, and the heart rate was closely monitored. Echocardiography measurements were performed by an echocardiogram system using a 17-MHZ transducer (Visual Sonic, Canada). On the parasternal short-axis view (PSAX) at the aortic level, Doppler-mode recording through the pulmonary artery was used to assess the pulmonary velocity profile. The time from the onset of pulmonary outflow to maximal flow (pulmonary artery acceleration time, PAAT) and the maximum pulmonary velocity $\left(\mathrm{PV}_{\max }\right)$ were measured.

\section{Right ventricular systolic pressure measurement}

Rats were anesthetized with pentobarbital sodium $(40 \mathrm{mg} / \mathrm{kg}$, ip). A polyethylene tube (outside diameter $=1 \mathrm{~mm}$ ) was inserted through the right jugular vein into the right ventricle (RV) to measure the right ventricle systolic pressure (RVSP). The RVSP was recorded using a BL-420S Data Acquisition \& Analysis System (Chengdu Tme Technology, China).

\section{Organ indices measurement and specimen preparation}

Once hemodynamic data were collected, the rats were euthanized, and the hearts and lungs were harvested. In some of the animals, the weights of the whole heart, right ventricular, lung and body were recorded. The ratios of right ventricleto-whole body weight and right ventricle-to-interventricular septal plus left ventricular weight (RV/LVS) were calculated.

\section{Determination of ALT, AST, LDH and NT-proBNP release into serum}

Myocardial cellular damage was evaluated by measuring the serum ALT, AST and LDH levels. At the end point, the serum ALT, AST and LDH activities were measured spectrophotometrically, and serum N-terminal pro brain natriuretic peptide (NT-proBNP) was quantified using commercial ELISA kits according to the manufacturer's instructions.

\section{ET-1 measurement by ELISA}

Lung tissues were homogenized and then centrifuged. The 
ET-1 levels in lung homogenates were measured using ELISA kits according to the manufacturer's instructions. The cytokine concentrations were quantified by reference to standard curves.

\section{Morphometric analysis}

The left lung, right ventricle and main pulmonary arteries were isolated, immediately flushed with saline and then fixed in $4 \%$ paraformaldehyde. Tissues were embedded in paraffin and sectioned at $5 \mu \mathrm{m}$. The right ventricle and main pulmonary arteries were stained with hematoxylin and eosin (H\&E). The left lung was stained with Masson's trichrome. The sections were examined under a light microscope for morphometric changes, and then photomicrographs were obtained. Only vessels exhibiting clearly defined external and internal elastic lamina were used in the analysis. The wall thickness of the main pulmonary artery, medial wall thickness to the external diameter (WT\%) of small pulmonary arteries and collagen disposition in lung were analyzed using the Image Pro Plus software, version 6.0. Briefly, \%MT was determined on the lung sections and calculated as follows: \%MT=[(external diameterinternal diameter)/external diameter] $\times 100$. For these studies, we evaluated vessels that had an external diameter between 20 and $150 \mu \mathrm{m}$. Pulmonary vessels from peribronchial or intraalveolar were used in this manuscript.

\section{Western blot analysis}

The left lung tissues were homogenized on ice in lysis buffer (containing $0.02 \mathrm{~mol} / \mathrm{L}$ Tris- $\mathrm{HCl}, 1 \%$ Triton $\mathrm{X}-100,0.15 \mathrm{~mol} / \mathrm{L}$ $\mathrm{NaCl}, 1 \mathrm{mmol} / \mathrm{L}$ ethylenediamine tetraacetic acid, $1 \mathrm{mmol} / \mathrm{L}$ EGTA, $2.5 \mathrm{mmol} / \mathrm{L}$ sodium pyrophosphate, $1 \mathrm{mmol} / \mathrm{L}$ $\beta$-glycerophosphate, and $1 \mathrm{mmol} / \mathrm{L}$ sodium orthovanadate) supplemented with a cocktail of protease inhibitors. The mixture was centrifuged at $12000 \mathrm{r} / \mathrm{min}$ for $5 \mathrm{~min}$ at $4^{\circ} \mathrm{C}$, and the supernatant was maintained at $-80^{\circ} \mathrm{C}$ until use. Protein concentration was determined spectrophotometrically using a bicinchoninic acid protein assay with serial dilution of bovine serum albumin (BSA) as the standard. Protein samples were mixed with $5 \times$ loading buffer and heated for $10 \mathrm{~min}$ at $95^{\circ} \mathrm{C}$.

Equal amounts $(50 \mu \mathrm{g})$ of protein extracts were subjected to SDS-PAGE and electrophoretically transferred to a polyvinylidene difluoride (PVDF) membrane. The membrane was blocked with $5 \%$ BSA for $1 \mathrm{~h}$ at room temperature and then incubated with primary antibodies (1:1000) overnight at $4{ }^{\circ} \mathrm{C}$. The membrane was then incubated with anti-rabbit IgG or anti-mouse IgG secondary antibodies (1:1000) for $2 \mathrm{~h}$ at $37^{\circ} \mathrm{C}$. Immunoreactivity bands were visualized by enhanced chemiluminescence and exposed to ChemiDoc-It Imaging System (UVP, USA). For quantification, ECL signals were digitized using Quantity One (Bio-rad, USA). Relative BMPRII and $\mathrm{p}$-Smad1/5 protein expression was normalized relative to GAPDH.

\section{Statistical analysis}

Statistical analysis was performed using SPSS software (SPSS 21.0, Chicago, USA). The results are expressed as the mean \pm SEM. All data were submitted to a one-way analysis of variance (ANOVA) followed by Dunnett's post hoc test. A $P$-value less than 0.05 was considered statistically significant.

\section{Results}

SAA alleviated MCT-induced increase in right ventricular systolic pressure

A significant increase in RVSP was noted in MCT-treated rats compared with the normal rats $(76.85 \pm 1.98$ vs $29.85 \pm 1.24$ $\mathrm{mmHg} ; \mathrm{P}<0.01)$. Treatment with three doses of SAA demonstrated a prominent reduction of RVSP (Figure 1A) to $60.97 \pm 2.34 \mathrm{mmHg}, 58.88 \pm 4.01 \mathrm{mmHg}$ and $56.19 \pm 2.23 \mathrm{mmHg}$ $(0.3,1,3 \mathrm{mg} / \mathrm{kg})$ from $76.85 \pm 1.98 \mathrm{mmHg}$ in the model group. No difference in heart rate was noted.

The PAAT and PVmax were used as echocardiographic indicators of pulmonary pressure and pulmonary vascular resistance measured invasively ${ }^{[22,23]}$. At the end point, the MCT group exhibited evidence of pulmonary hypertension with a reduction in PAAT and $\mathrm{PV}_{\max }$ compared with the control group. Although the bosentan group and SAA groups exhibited evidence of pulmonary hypertension, the extent was less severe compared with the model group.

\section{Effect of SAA treatment on organ indices of PAH rats}

The weight ratio of RV/LVS is typically calculated as an indicator of right ventricular hypertrophy (RVH). MCT caused a significant increase in both the right ventricle/body weight ratio $(1.00 \pm 0.06$ vs $0.29 \pm 0.01 \mathrm{mg} / \mathrm{g} ; P<0.01)$ and the right ventricle/left ventricle plus septum (RV/LVS) ratio $(0.31 \pm 0.01$ vs $0.13 \pm 0.003 \mathrm{mg} / \mathrm{mg} ; P<0.01)$.

Treatment with SAA $(0.3,1,3 \mathrm{mg} / \mathrm{kg})$ significantly decreased the $\mathrm{RV} /$ body weight ratio from $1.00 \pm 0.06$ (model group) to $0.68 \pm 0.02(P<0.01), 0.66 \pm 0.01(P<0.01)$, and $0.63 \pm 0.03$ $(P<0.01)$, respectively (Figure $2 \mathrm{~B})$. SAA treatment also decreased the RV/LVS ratio from $0.31 \pm 0.01$ (model group) to $0.21 \pm 0.01(P<0.01), 0.22 \pm 0.01(P<0.01)$, and $0.23 \pm 0.004(P<0.01)$, respectively (Figure $2 \mathrm{C}$ ).

Similarly, the lung weight/body weight ratio was significantly increased in the model group $(8.85 \pm 0.37)$ compared with the control group $(3.99 \pm 0.09 ; P<0.01$, Figure 2D). The SAA groups exhibited a significant decrease in this ratio, ie, to $6.33 \pm 0.27,5.94 \pm 0.30$ and $6.43 \pm 0.21$, respectively, compared with the model group.

\section{SAA alleviated vascular remodeling induced by MCT injection}

Remodeling of small pulmonary arteries (peribronchial or intra-alveolar vessels) was determined by Masson trichrome stain (Figure 3A, 3B). The medial wall thickness of small pulmonary arteries in the model group was significantly increased. Similarly, compared with the control group, an incrassation in the main pulmonary artery was observed at the end point, which was marked by significant increases in medial thickness (Figure 3C). SAA administration reversed the pulmonary artery remodeling in a dose-dependent manner (Figure 3). 
A

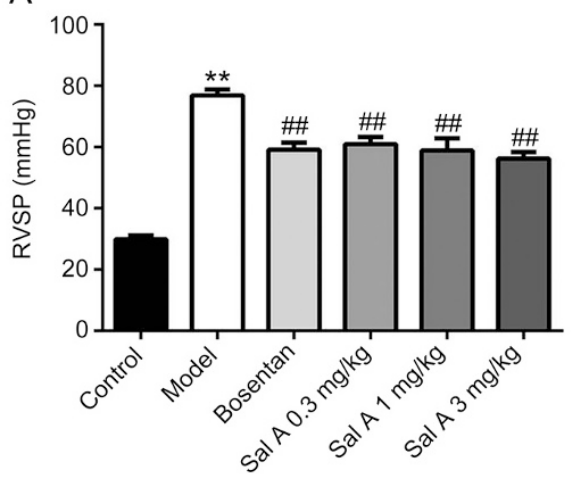

B

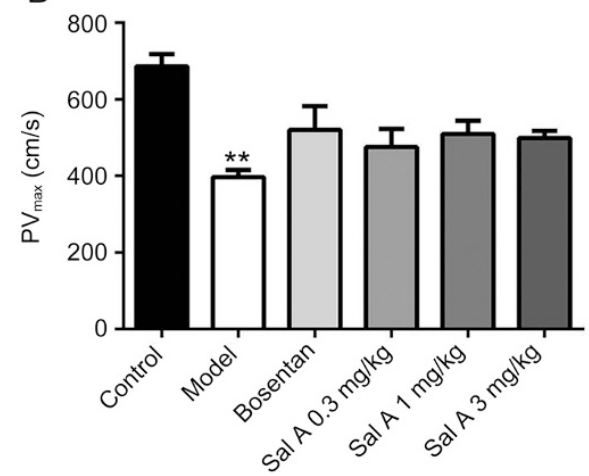

C

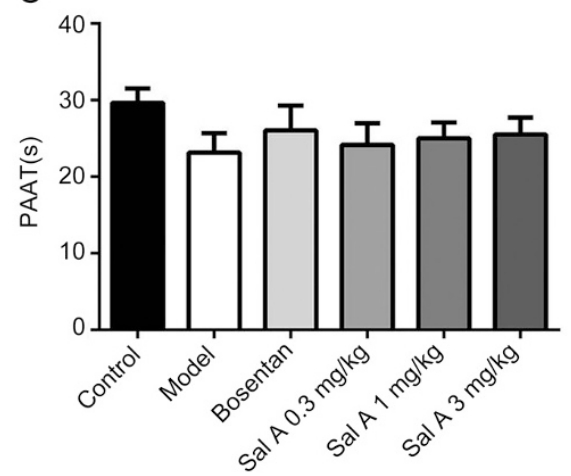

D
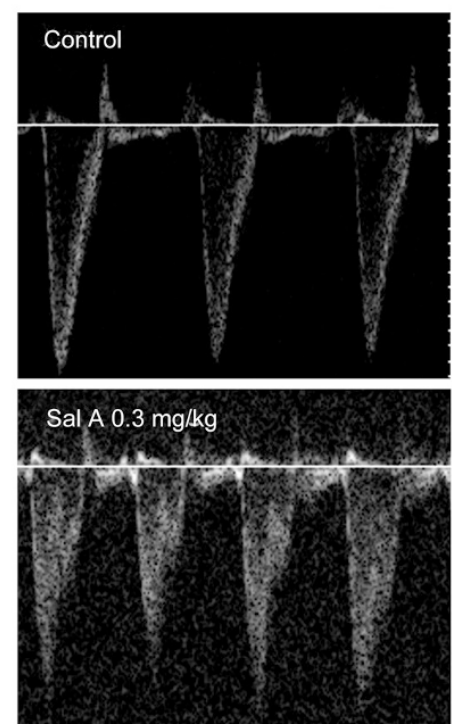
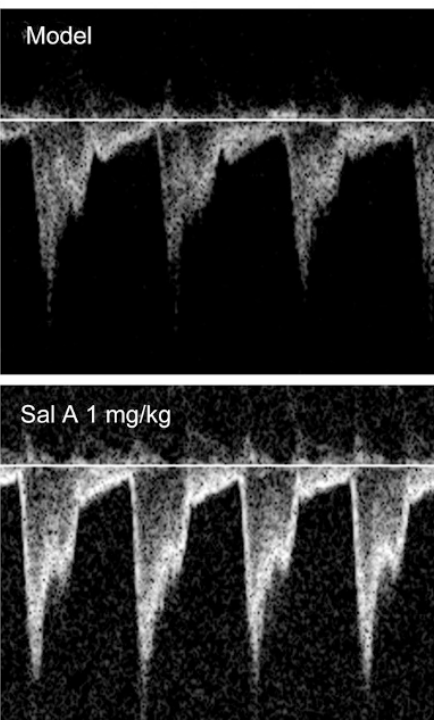

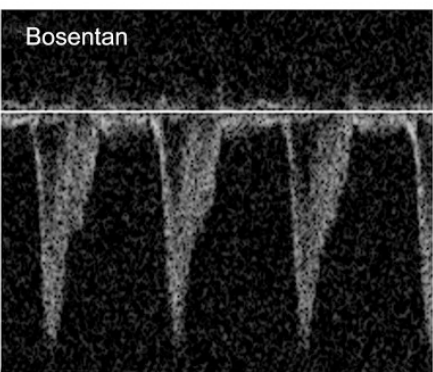

Sal A 3 mglikg

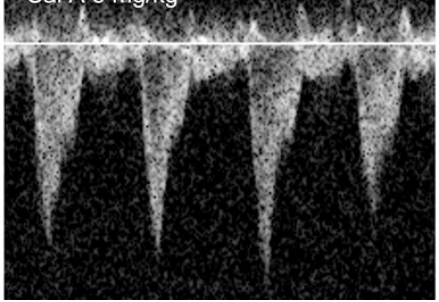

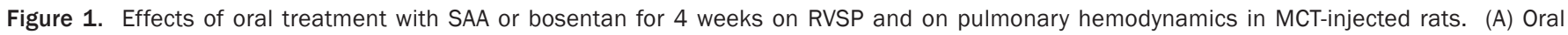

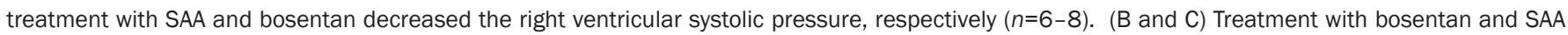

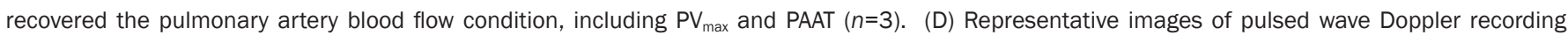

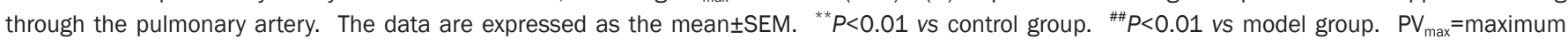
pulmonary velocity; PAAT=pulmonary artery acceleration time.

\section{SAA treatment blunted myocardial injury in response to pressure} overload

Myocyte morphology of right ventricular in the model group shows disarranged cell bundles, and the change was alleviated in the bosentan- or SAA-treated groups (Figure 4A). Circulating levels of NT-proBNP may correlate with PAH clinical severity. To evaluate the degree of myocardial hypertrophy, serum NT-proBNP was tested. The administration of SAA caused a significant decrease in the serum NT-proBNP levels compared with MCT-treated rats. Myocardial cellular damage was evaluated by the detection of the serum AST, ALT and LDH levels. At the end point, the serum AST, ALT and LDH activities were measured spectrophotometrically. Here, $3 \mathrm{mg} /$ $\mathrm{kg}$ SAA decreased the serum AST and ALT levels compared with the model group.
SAA attenuated MCT-induced lung mesenchymal damage and ET-1 expression

Histological alterations in MCT-induced lung damage and collagen deposition in MCT group are demonstrated in Figure 5A. The normal structure of alveoli was lost in many areas. These maladaptive changes were alleviated in the bosentan- or SAA-treated groups. Masson trichrome staining also revealed obvious collagen deposition (yellow arrows) in the pulmonary interstitium in the model group. MCT-induced lung interstitial fibrosis was significantly alleviated in the bosentan- and SAA-treated groups (Figure 5B). The release of the ET-1 was increased to $37.14 \pm 6.24(P<0.01)$ in lungs of the model group compared with the control group. The bosentan and SAA groups exhibited a significant down-regulation of ET-1 secretion compared with MCT-treated rats $(P<0.01$; Figure $5 C)$. 


\section{A}
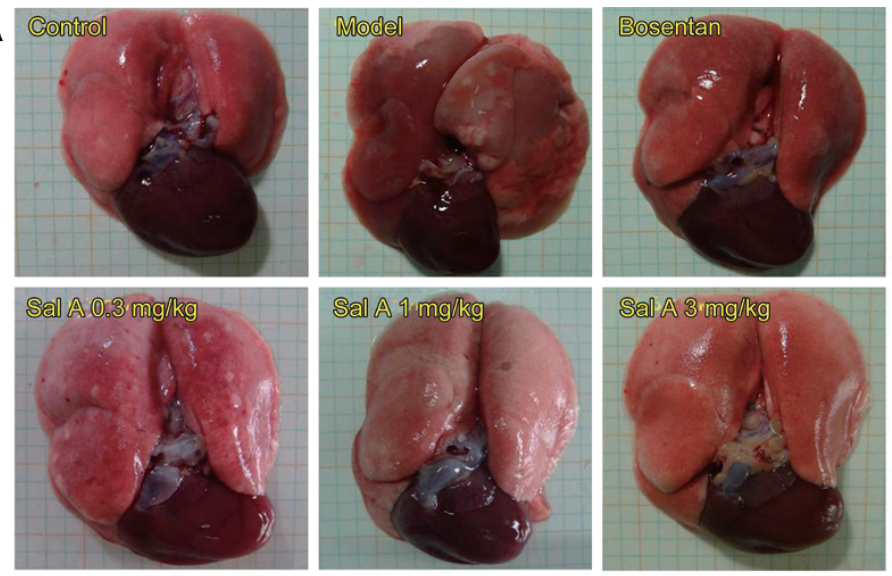

C

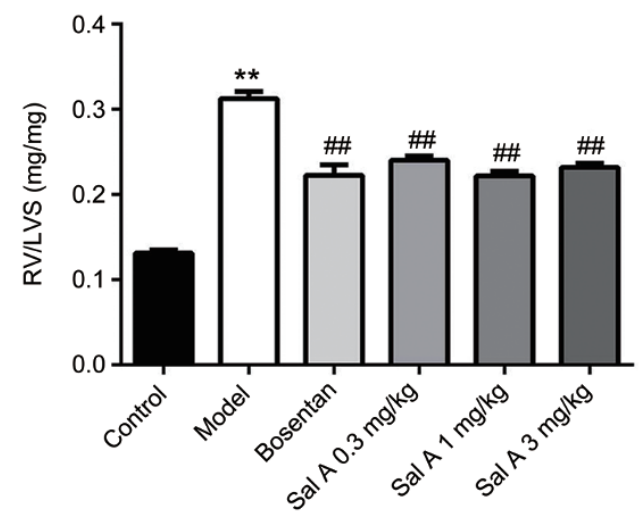

B
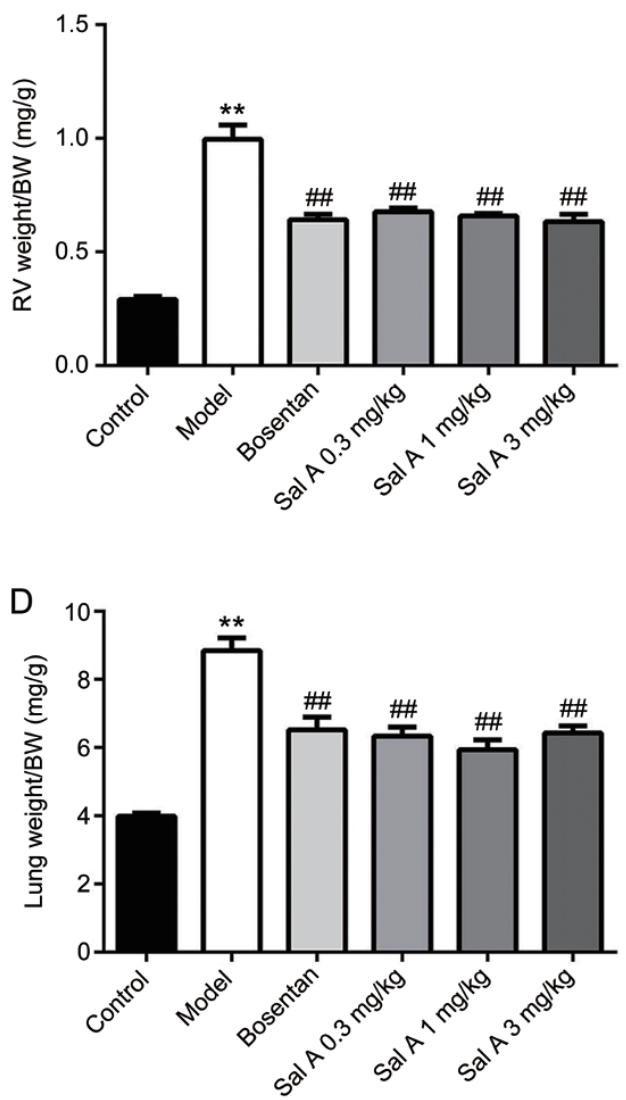

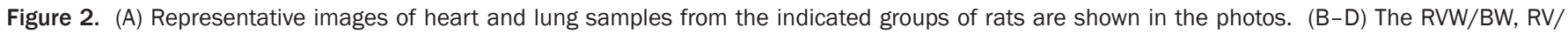

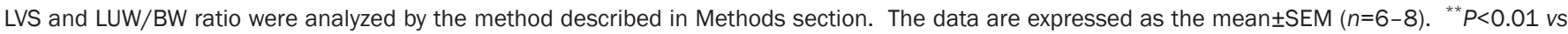
control group. ${ }^{\# \#} P<0.01$ vs model group. BW=body weight; RV=right ventricle; LVS=left ventricle and septum.

\section{SAA inhibited apoptosis in lung}

The expression of Bax protein as an index of apoptosis was increased in the model group compared with the control group in lung tissues (Figure 6A). Bax protein levels were decreased in the SAA-treated groups, but no statistical significance was noted between these groups.

By contrast, Bcl-2 protein expression (Figure 6B) in lung parenchyma as an indicator of anti-apoptosis exhibited an opposite effect compared with that of apoptotic biomarkers among the five groups. Furthermore, the Bax/Bcl-2 ratio increased in the model group compared with control group, and the tendency could be reversed by SAA $1 \mathrm{mg} / \mathrm{kg}$ treatment.

\section{SAA restored defective bone morphogenetic protein signaling in MCT-exposed rat lung}

BMPRII protein expression was reduced in lungs of MCTexposed rats, consistent with previous reports ${ }^{[2]}$. To investigate the influence of SAA on the BMPR-Smad1/5 pathway, we assessed the BMPRII and P-Smad $1 / 5$ protein levels in the lung tissue of control, model, bosentan and SAA $(0.3,1,3 \mathrm{mg} / \mathrm{kg})$ groups by Western blot analysis. The results indicated that
MCT treatment reduced BMPRII $(0.62 \pm 0.06, P<0.05)$ protein expression. The SAA groups exhibited an increase in BMPRII expression, but no significant difference was observed. Similarly, rats treated with MCT exhibited a significant downregulation of p-Smad $1 / 5$ in lungs. SAA $3 \mathrm{mg} / \mathrm{kg}$ treatment increased Smad1/ 5 phosphorylation in lung.

\section{Discussion}

PAH is a multifactorial and progressive disease that results in high morbidity and mortality ${ }^{[25-27]}$. Salvia miltiorrhiza has been used in clinics in China for thousands of years to treat heart failure with little side effects. As the main component of Salvia miltiorrhiza, SAA holds significant potential for the treatment of cardiovascular diseases. The current study, which investigated the impact of SAA on MCT-induced PAH, yielded several striking implications. First, MCT treatment elicited pulmonary artery remodeling, pulmonary hemodynamic abnormalities and increases in the right ventricular systolic pressure. These changes could be effectively attenuated by SAA treatment. Second, SAA treatment effectively reduced MCT-induced lung parenchymal injury and collagen deposition, thereby protecting the myocardium against hyper- 
A
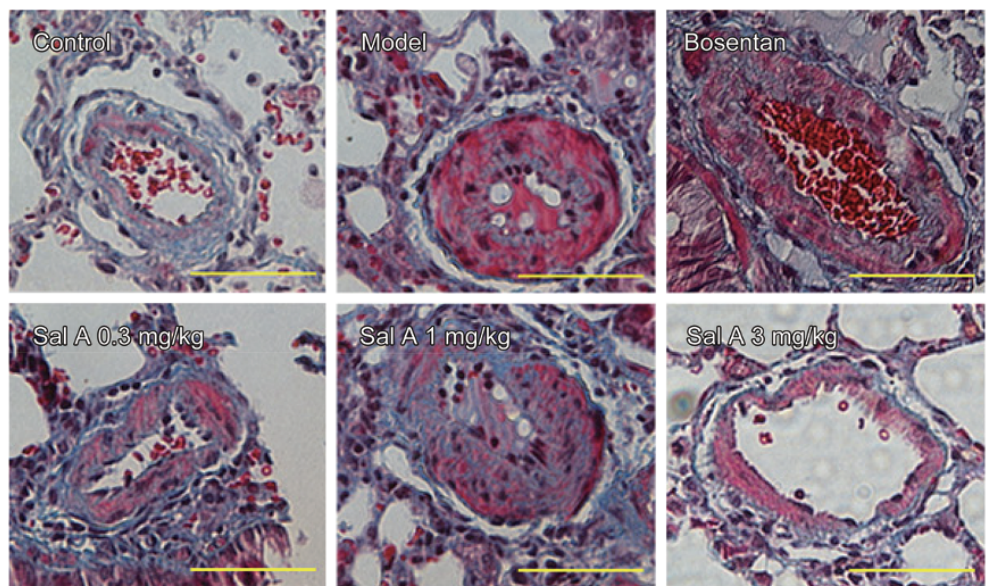

B
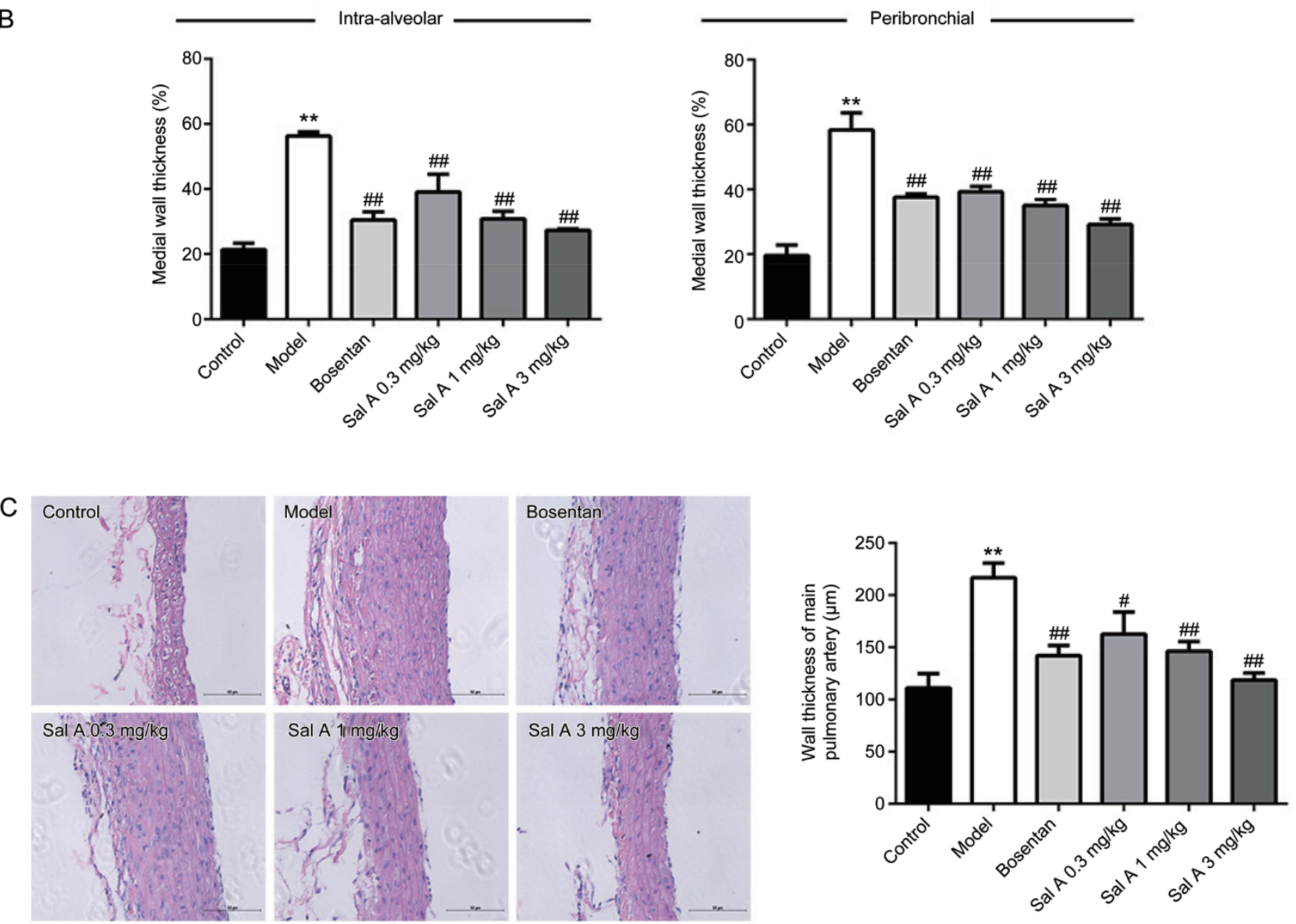

Figure 3. (A) Representative images of Masson staining of the small pulmonary arteries. The original magnification of the images was $\times 400$ (yellow arrows). Each bar represents $20 \mu \mathrm{m}$. (B) Medial wall thickness of small pulmonary arteries (peribronchial and intra-alveolar vessels). (C) Representative photos of H\&E staining of the main pulmonary arteries. The original magnification of the images was $\times 200$. (D) Wall thickness of main pulmonary artery. The data are expressed as the mean \pm SEM $(n=3-5) .{ }^{* *} P<0.01$ vs control group. ${ }^{\#} P<0.05,{ }^{\# \#} P<0.01$ vs model group.

trophic damage. Furthermore, SAA treatment attenuated the process of apoptosis and fibrosis in lung after MCT injection. Moreover, SAA therapy up-regulated BMPRII expression and
Smad1/5 phosphorylation in pulmonary tissues, thus implying its possible anti-fibrotic role in the PAH model.

Fibromuscular hypertrophy and hyperplasia in the arterial 
A
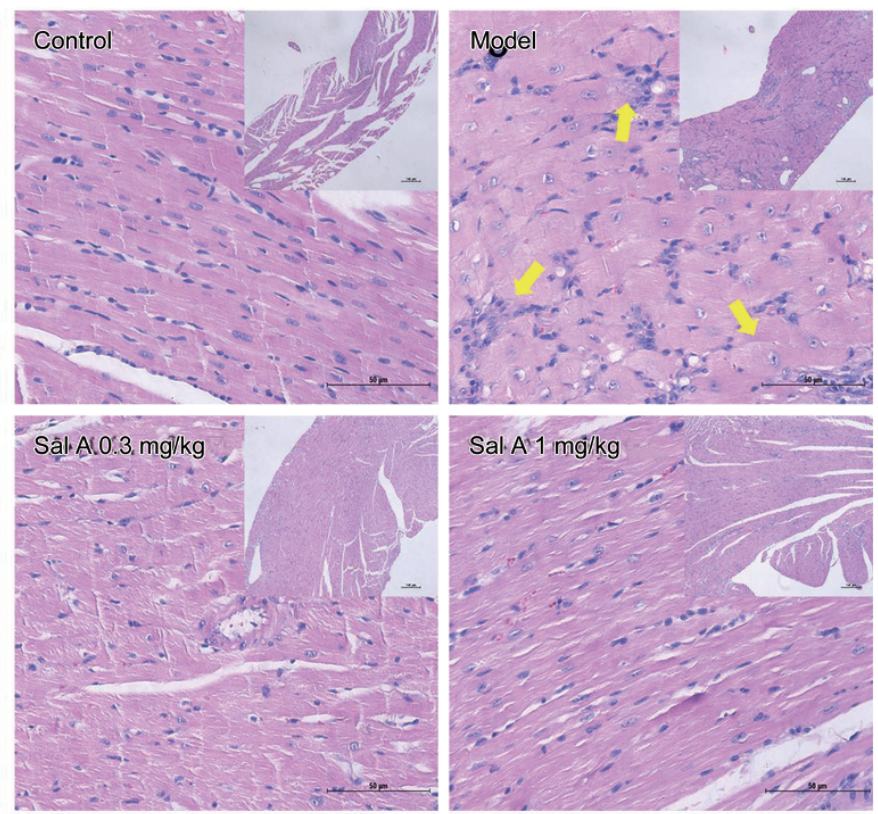
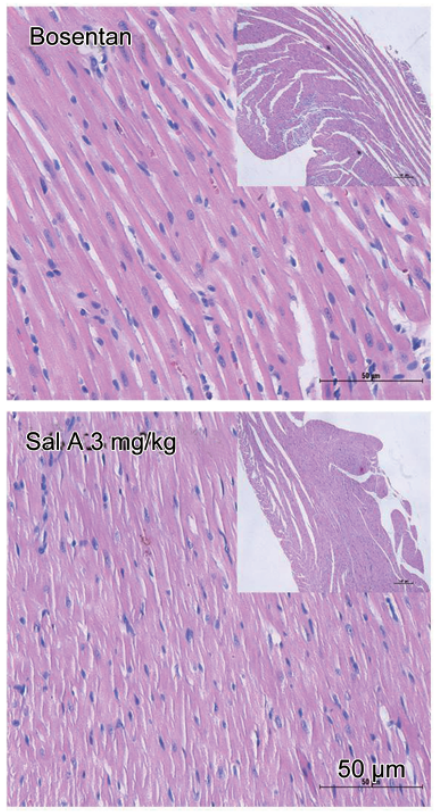

B
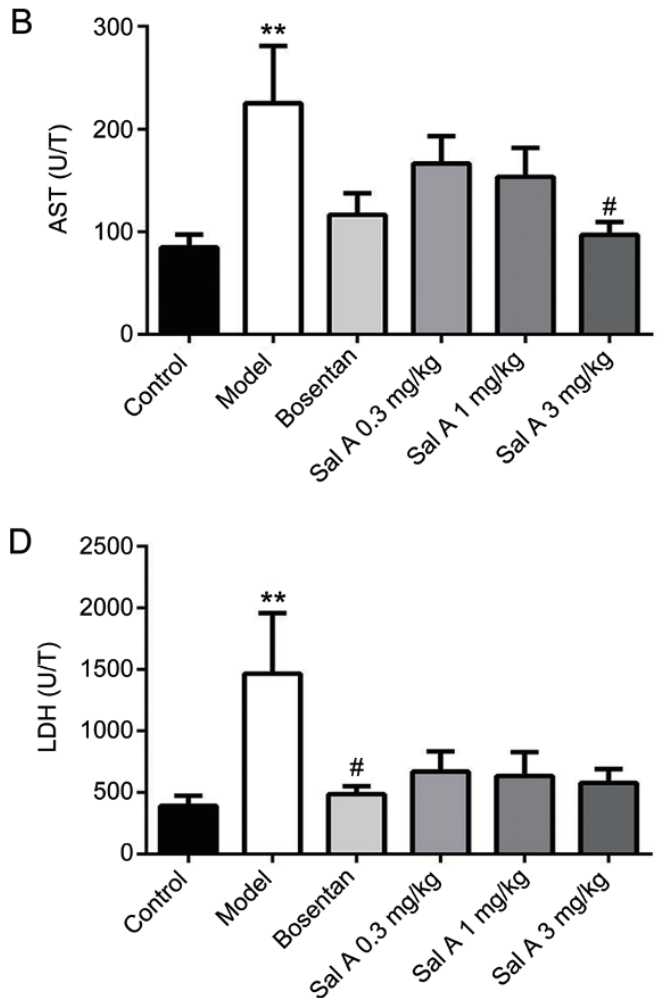

C

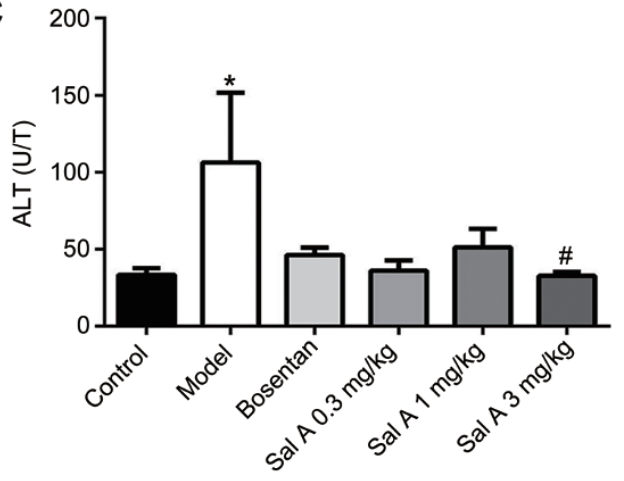

E

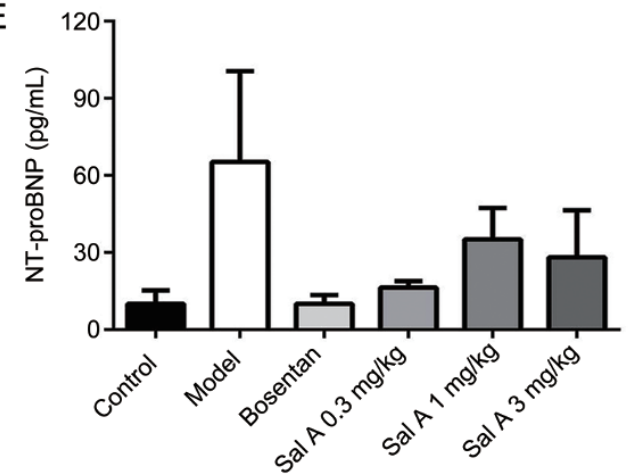

Figure 4. SAA treatment blunted myocardial injury in response to pressure-overload. (A) H\&E staining shows myocyte morphology of right ventricle in the control, model, bosentan, and SAA $(0.3,1,3 \mathrm{mg} / \mathrm{kg})$-treated groups. The original magnification of the big images was $\times 200$, and that of small images was $\times 40$. Arrows indicate cellular hypertrophy and disarranged cell bundles. (B-E) SAA and bosentan decreased serum level of AST, ALT, LDT, and NT-proBNP in PAH rats. Data are shown as mean \pm SEM $(n=6) .{ }^{*} P<0.05,{ }^{* *} P<0.01$ vs control group. ${ }^{\#} P<0.05$ vs model group.

wall of PAH rats increased RVSP and induced RV hypertrophy, as indicated by the RV/BW or RV/LVS ratio. Daily oral treatment with SAA after MCT-injection abolished the increase in RVSP and reduced RV hypertrophy. These benefi- cial effects might be caused by decreased blood resistance and pulmonary vascular remodeling and a consequent reduction in RV systolic work.

Echocardiography is the most common and convenient 
A
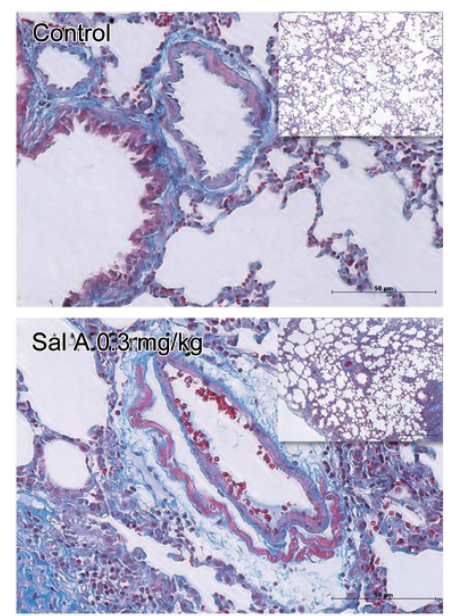
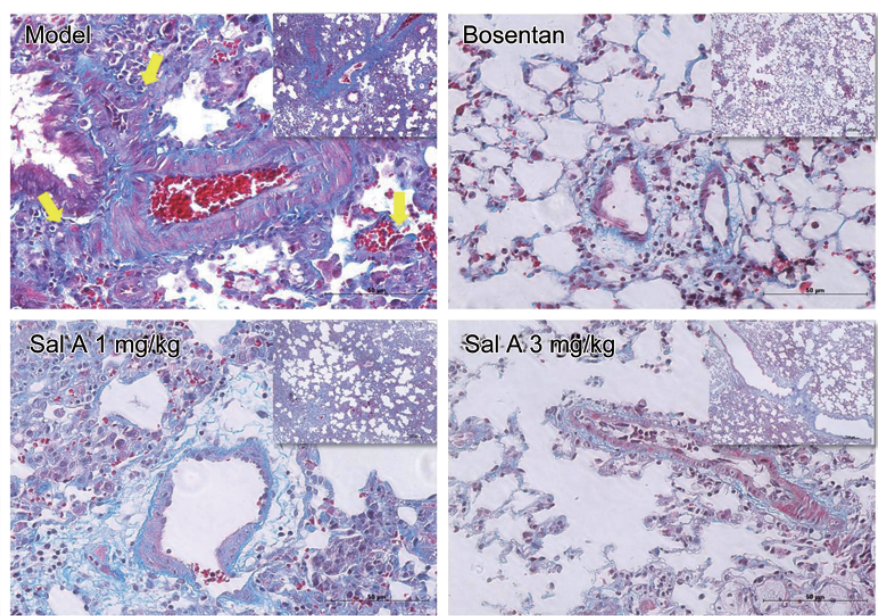

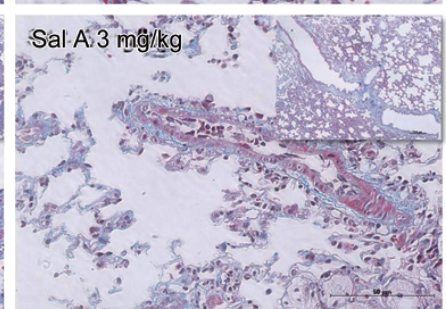

B

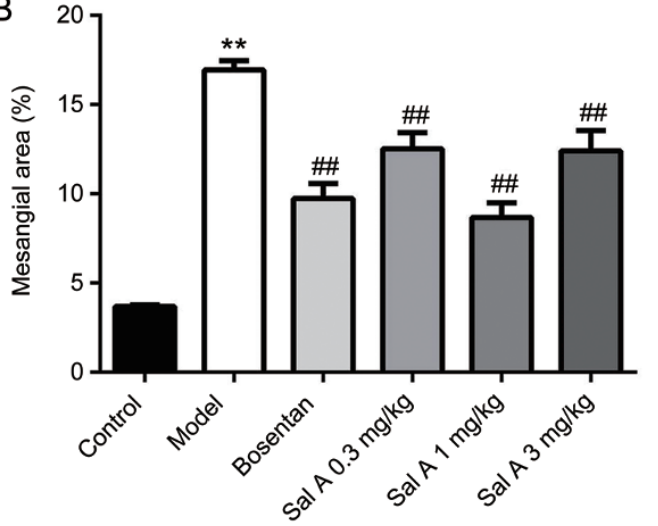

C

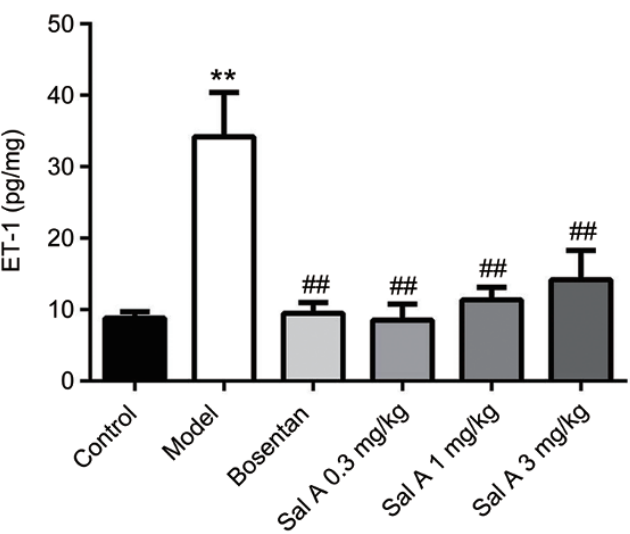

Figure 5. (A) Representative images of Masson's trichrome-stained lungs from the indicated groups of rats are shown. The original magnification of the big images was $\times 200$, and that of small images was $\times 40$. (B) The relative mesangial area $(n=3-4)$ and $(C)$ secretion of the ET-1 ( $n=6)$ were analyzed by the method described in Methods section. The data are expressed as the mean \pm SEM. ${ }^{* *} P<0.01$ vs control group. ${ }^{\# \#} P<0.01$ vs model group.

method used to evaluate RV function and pulmonary circulation hemodynamics ${ }^{[28,29]}$. Several echocardiographic parameters have been used as reliable indicators in previous studies, including PAAT and $\mathrm{PV}_{\max }{ }^{[30,31]}$. In this study, echocardiographic analysis of the MCT-exposed rats revealed a reduction of the $\mathrm{PV}_{\max }$ and PAAT due to the hypertrophy and stiffness of the pulmonary artery. Treatment with SAA normalized these changes in rats.

It is thought that pathological vascular remodeling plays a crucial role in pulmonary arterial hypertension. The accumulation of extracellular matrix, including collagen, as well as vascular smooth muscle cell proliferation and hypertrophy contribute to medial hypertrophy and muscularization, which lead to the obliteration of pulmonary arteries and the sustained elevation of pulmonary arterial pressure ${ }^{[32,36]}$. Monocrotaline is metabolized to monocrotaline pyrrole in the liver. The metabolite injures the pulmonary arterial endothelium and evokes pulmonary artery remodeling ${ }^{[3,38]}$. Thickening of the small artery wall and pulmonary artery stenosis leads to irreversible pulmonary hypertension. Large artery remodeling is characterized by an increase in intima-media thickness
(IMT) (approximately 15\%-40\%) ${ }^{[36]}$. In the present study, obviously thickened small pulmonary arteries and pulmonary trunk could be observed after MCT injection, and this finding is consistent with the pathological process of PAH. Our findings demonstrated that SAA treatment significantly attenuated wall thickening in pulmonary arteries induced by MCT.

Apoptosis from lung parenchymal damage has been well recognized in the setting of MCT-induced PAH in recent studies $^{[38,39]}$. Consistent with the findings of previous reports ${ }^{[39]}$, we demonstrate that the expression of apoptotic biomarkers was substantially increased in lungs of MCT-treated animals. The anti-apoptotic effects of SAA might contribute to inhibition of the development of pulmonary arterial hypertension.

BMPRII mutations lead to a loss of the growth-suppressive effects of BMPs in pulmonary artery smooth muscle cells via a reduction in the phosphorylation of the downstream effector Smad1/5, which is a major target protein in response to BMP signaling ${ }^{[7]}$. In the lung, BMPRII is highly expressed on the vascular endothelium of the pulmonary arteries ${ }^{[40]}$. BMPRII expression is markedly reduced in the pulmonary arteries of patients with mutations in the BMPRII gene. Smad1/5 
A
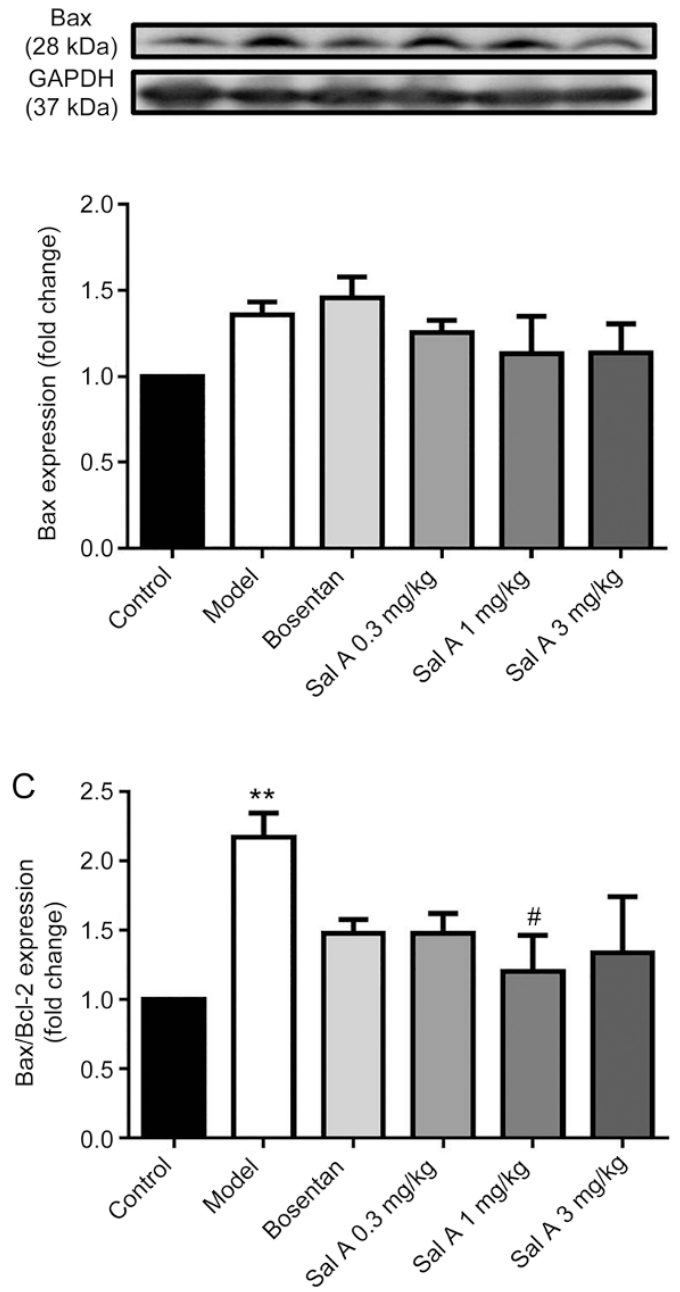

B
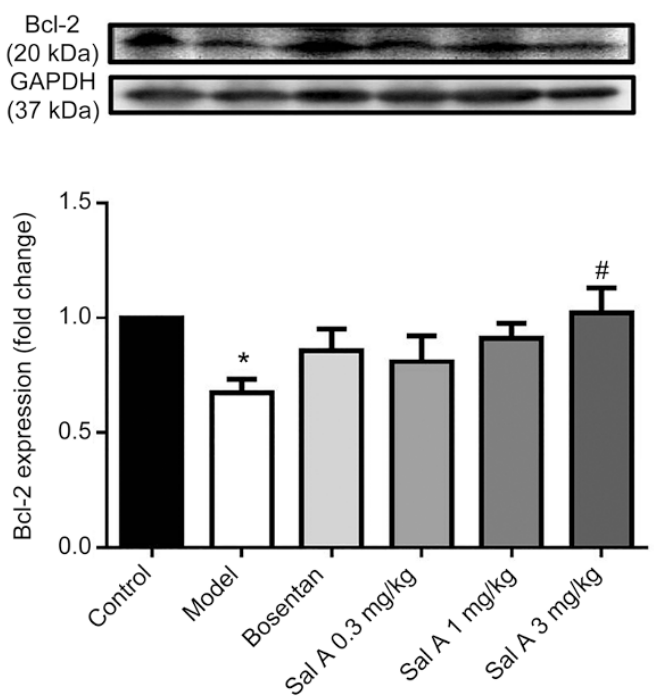

Figure 6. SAA treatment attenuated MCT-induced increases in markers of apoptosis. (A and B) Protein isolates of left lung from control, model, bosentan and SAA $(0.3,1,3 \mathrm{mg} / \mathrm{kg})$ treated groups were analyzed by immunoblotting to determine the amount of Bax and Bcl-2 and normalized to the expression of GAPDH. (C) Normalized band intensity quantification shows the Bax/Bcl-2 ratio. The data are expressed as the mean \pm SEM ( $n=4$ ). ${ }^{*} P<0.05,{ }^{* *} P<0.01$ vs control group. ${ }^{\#} P<0.05$ vs model group.

phosphorylation is also reduced in the pulmonary arteries of patients with underlying BMPRII mutations and in patients with IPAH with no identifiable mutation ${ }^{[41]}$. In the current study, a slight restoration of BMPRII was observed in SAAtreated groups compared with the model group. Additionally, our data revealed that Smad1/5 phosphorylation increased upon SAA treatment. In addition, the BMP-Smad pathway potentially influences the release of ET-1 and modifies its contractile effect on human pulmonary vascular smooth muscle cells $^{[42]}$, which is consistent with the decrease in ET-1 levels observed in lung in the present study.

Taken together, the results demonstrate that SAA could prevent the development of PAH in a MCT-induced rat model and alleviate right ventricular hypertrophy, myocardial injury and pulmonary vascular remodeling. Moreover, activation of the BMPR-Smad signal pathway and inhibition of apoptosis in lung might serve as mechanisms that are involved in the therapeutic effects of SAA. Therefore, these data imply that SAA may be an attractive molecule for developing drugs against $\mathrm{PAH}$.

\section{Acknowledgements}

This study was supported by grants from the National Natural Science Foundation of China (№ 81573645 and 81202538) and the National Science and Technology Major Project (№ 2013ZX09103001-008).

\section{Author contribution}

Yu-cai CHEN designed the experiments, drafted the manuscript and conducted experiments; Tian-yi YUAN and Huifang ZHANG prepared reagents and conducted experiments; Dan-shu WANG and Yu YAN participated in the Western blotting assay; Zi-ran NIU and Yi-huang LIN performed data analysis; Guan-hua DU and Lian-hua FANG participated in 
A
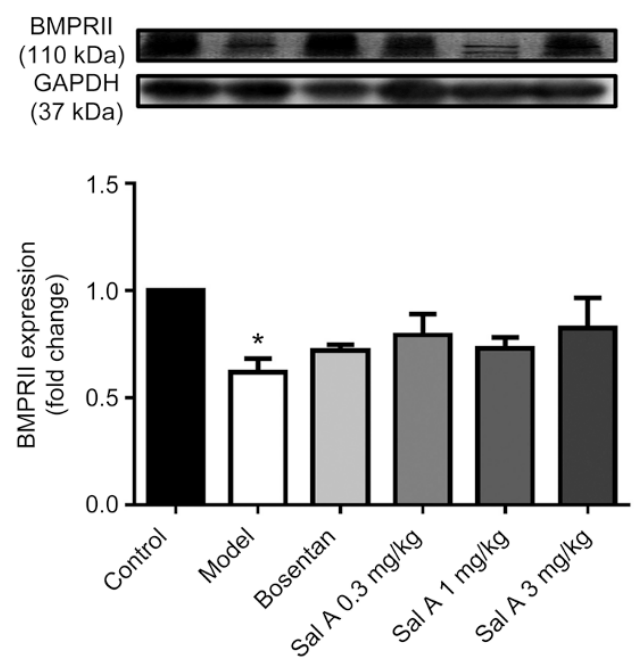

B

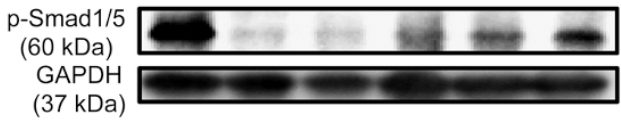

(37 kDa)

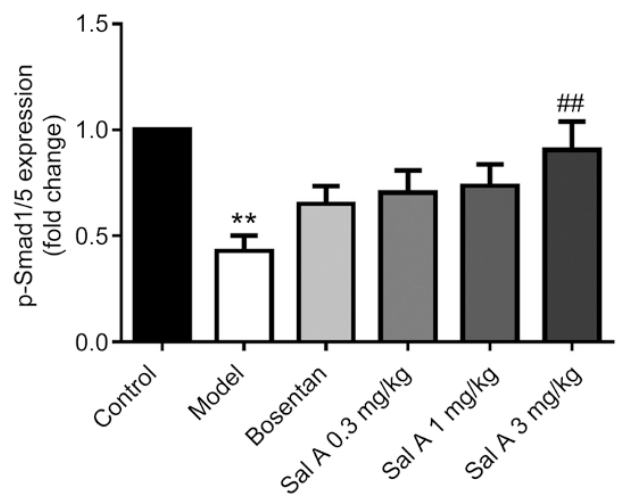

Figure 7. Western blot analysis for BMPRII-Smad pathway in lung parenchyma at d 28 after MCT administration. (A and B) Protein isolates of left lung from control, model, bosentan and SAA $(0.3,1,3 \mathrm{mg} / \mathrm{kg})$ treated groups were analyzed by immunoblotting to determine the amount of BMPRII and p-Smad1/5, and normalized to the expression of GAPDH ( $n=4) .{ }^{*} P<0.05,{ }^{* *} P<0.01$ vs control group. ${ }^{\# \#} P<0.01$ vs model group.

experimental design and critically revised the manuscript for important intellectual content.

\section{References}

1 McLaughlin VV. Looking to the future: a new decade of pulmonary arterial hypertension therapy. Eur Respir J 2011; 20: 262-9.

2 Mathew R. Pathogenesis of pulmonary hypertension: a case for caveolin-1 and cell membrane integrity. Am J Physiol-Heart C 2014; 306: H15-25.

3 Rosanio S, Pelliccia F, Gaudio C, Greco C, Keylani AM, D’Agostino DC. Pulmonary arterial hypertension in adults: novel drugs and catheter ablation techniques show promise? Biomed Res Int 2014; 2014: 743868

4 Zamanian RT, Kudelko KT, Sung YK, de Jesus Perez V, Liu J, Spiekerkoetter E. Current clinical management of pulmonary arterial hypertension. Circ Res 2014; 115: 131-47.

5 Han C, Hong KH, Kim YH, Kim MJ, Song C, Kim MJ, et al. SMAD1 deficiency in either endothelial or smooth muscle cells can predispose mice to pulmonary hypertension. Hypertension 2013; 61: 1044-52.

6 Upton PD, Davies RJ, Tajsic T, Morrell NW. Transforming growth factorbeta(1) represses bone morphogenetic protein-mediated Smad signaling in pulmonary artery smooth muscle cells via Smad3. Am J Resp Cell Mol 2013; 49: 1135-45.

7 Yang J, Davies RJ, Southwood M, Long L, Yang X, Sobolewski A, et al. Mutations in bone morphogenetic protein type II receptor cause dysregulation of Id gene expression in pulmonary artery smooth muscle cells: implications for familial pulmonary arterial hypertension. Circ Res 2008; 102: 1212-21.

8 Nishikawa-Takahashi M, Ueno S, Kario K. Long-term advanced therapy with bosentan improves symptoms and prevents deterioration of inoperable chronic thromboembolic pulmonary hypertension. Life Sci 2014; 118: 410-3.

9 Fontoura D, Oliveira-Pinto J, Tavares-Silva M, Leite S, Vasques-Novoa F, Mendes-Ferreira P, et al. Myocardial and anti-inflammatory effects of chronic bosentan therapy in monocrotaline-induced pulmonary hypertension. Rev Port Cardiol 2014; 33: 213-22.
10 Gong S, Peng L, Yan B, Dong Q, Seng Z, Wang W, et al. Bosentan reduces neuronal apoptosis following spinal cord ischemic reperfusion injury. Spinal Cord 2014; 52: 181-5.

11 Lee FY, Lu HI, Zhen YY, Leu S, Chen YL, Tsai TH, et al. Benefit of combined therapy with nicorandil and colchicine in preventing monocrotaline-induced rat pulmonary arterial hypertension. Eur J Pharm Sci 2013; 50: 372-84.

12 Li L, Wei C, Kim IK, Janssen-Heininger Y, Gupta S. Inhibition of nuclear factor-kappaB in the lungs prevents monocrotaline-induced pulmonary hypertension in mice. Hypertension 2014; 63: 1260-9.

13 Xu T, Wu X, Chen Q, Zhu S, Liu Y, Pan D, et al. The anti-apoptotic and cardioprotective effects of salvianolic acid $\mathrm{A}$ on rat cardiomyocytes following ischemia/reperfusion by DUSP-mediated regulation of the ERK1/2/JNK pathway. PloS One 2014; 9: e102292.

$14 \mathrm{Ho} \mathrm{JH}$, Hong CY. Salvianolic acids: small compounds with multiple mechanisms for cardiovascular protection. J Biomed Sci 2011; 18: 30.

15 Jiang B, Li D, Deng Y, Teng F, Chen J, Xue S, et al. Salvianolic acid $A$, a novel matrix metalloproteinase-9 inhibitor, prevents cardiac remodeling in spontaneously hypertensive rats. PloS One 2013; 8: e59621.

16 Teng F, Yin Y, Cui Y, Deng Y, Li D, Cho K, et al. Salvianolic acid $A$ inhibits endothelial dysfunction and vascular remodeling in spontaneously hypertensive rats. Life Sci 2016; 144: 86-93.

17 Qiang GF, Yang XY, Xuan Q, Shi LL, Zhang HA, Chen BN, et al. Salvianolic acid A prevents the pathological progression of hepatic fibrosis in high-fat diet-fed and streptozotocin-induced diabetic rats. Am J Chin Med 2014; 5: 1183-98.

18 Ahmed LA, Obaid AA, Zaki HF, Agha AM. Naringenin adds to the protective effect of $L$-arginine in monocrotaline-induced pulmonary hypertension in rats: favorable modulation of oxidative stress, inflammation and nitric oxide. Eur J Pharm Sci 2014; 62: 161-70.

19 Haga S, Tsuchiya H, Hirai T, Hamano T, Mimori A, Ishizaka Y. A novel ACE2 activator reduces monocrotaline-induced pulmonary hypertension by suppressing the JAK/STAT and TGF-beta cascades with restored caveolin-1 expression. Exp Lung Res 2015; 41: 21-31. 
20 Rudski LG, Lai WW, Afilalo J, Hua L, Handschumacher MD, Chandrasekaran $\mathrm{K}$, et al. Guidelines for the echocardiographic assessment of the right heart in adults: a report from the American Society of Echocardiography endorsed by the European Association of Echocardiography, a registered branch of the European Society of Cardiology, and the Canadian Society of Echocardiography. J Am Soc Echocardiog 2010; 23: 685-713.

21 Cheng HW, Fisch S, Cheng S, Bauer M, Ngoy S, Qiu Y, et al. Assessment of right ventricular structure and function in mouse model of pulmonary artery constriction by transthoracic echocardiography. Jove-J Vis Exp 2014; 84: e51041.

22 Isobe M, Yazaki Y, Takaku F, Koizumi K, Hara K, Tsuneyoshi H, et al. Prediction of pulmonary arterial pressure in adults by pulsed Doppler echocardiography. Am J Cardiol 1986; 57: 316-21.

23 Jones JE, Mendes L, Rudd MA, Russo G, Loscalzo J, Zhang YY. Serial noninvasive assessment of progressive pulmonary hypertension in a rat model. Am J Physiol-Heart C 2002; 283: H364-71.

24 Long L, Crosby A, Yang X, Southwood M, Upton PD, Kim DK, et al. Altered bone morphogenetic protein and transforming growth factorbeta signaling in rat models of pulmonary hypertension: potential for activin receptor-like kinase- 5 inhibition in prevention and progression of disease. Circulation 2009; 119: 566-76.

25 Paulin R, Michelakis ED. The metabolic theory of pulmonary arterial hypertension. Circ Res 2014; 115: n148-64.

26 Rabinovitch M, Guignabert C, Humbert M, Nicolls MR. Inflammation and immunity in the pathogenesis of pulmonary arterial hypertension. Circ Res 2014; 115: 165-75.

27 Jasinska-Stroschein M, Orszulak-Michalak D. The current approach into signaling pathways in pulmonary arterial hypertension and their implication in novel therapeutic strategies. Pharmacol Rep 2014; 66: 552-64.

28 Thibault HB, Kurtz B, Raher MJ, Shaik RS, Waxman A, Derumeaux G, et al. Noninvasive assessment of murine pulmonary arterial pressure: validation and application to models of pulmonary hypertension. Circ-Cardiovasc Imag 2010; 3: 157-63.

29 Yang T, Liang Y, Zhang Y, Gu Q, Chen G, Ni XH, et al. Echocardiographic parameters in patients with pulmonary arterial hypertension: correlations with right ventricular ejection fraction derived from cardiac magnetic resonance and hemodynamics. PLoS One 2013;8: e71276.

30 Bossone E, D'Andrea A, D'Alto M, Citro R, Argiento P, Ferrara F, et al. Echocardiography in pulmonary arterial hypertension: from diagnosis to prognosis. J Am Soc Echocardiog 2013; 26: 1-14.
31 Temple IP, Monfredi O, Quigley G, Schneider H, Zi M, Cartwright EJ, et al. Macitentan treatment retards the progression of established pulmonary arterial hypertension in an animal model. Int J Cardiol 2014; 177: 423-8.

32 Stenmark KR, Meyrick B, Galie N, Mooi WJ, McMurtry IF. Animal models of pulmonary arterial hypertension: the hope for etiological discovery and pharmacological cure. Am J Physiol-Lung C 2009; 297: L1013-32.

33 Rabinovitch M. Pathobiology of pulmonary hypertension. Annu Rev Pathol 2007; 2: 369-99.

34 Spiekerkoetter E, Tian X, Cai J, Hopper RK, Sudheendra D, Li CG, et al. FK506 activates BMPR2, rescues endothelial dysfunction, and reverses pulmonary hypertension. J Clin Invest 2013; 123: 3600-13.

35 Okada M, Harada T, Kikuzuki R, Yamawaki H, Hara Y. Effects of telmisartan on right ventricular remodeling induced by monocrotaline in rats. J Pharmacol Sci 2009; 111: 193-200.

36 Laurent S, Boutouyrie P. The structural factor of hypertension: large and small artery alterations. Circ Res 2015; 116: 1007-21.

37 Sun CK, Lee FY, Sheu JJ, Yuen CM, Chua S, Chung SY, et al. Early combined treatment with cilostazol and bone marrow-derived endothelial progenitor cells markedly attenuates pulmonary arterial hypertension in rats. J Pharmacol Exp Ther 2009; 330: 718-26.

38 Yen CH, Leu S, Lin YC, Kao YH, Chang LT, Chua S, et al. Sildenafil limits monocrotaline-induced pulmonary hypertension in rats through suppression of pulmonary vascular remodeling. J Cardiovasc Pharm 2010; 55: 574-84.

39 Xie P, Duan Y, Guo X, Hu L, Yu M. SalA attenuates hypoxiainduced endothelial endoplasmic reticulum stress and apoptosis via downregulation of VLDL receptor expression. Cell Physiol Biochem 2015; 35: 17-28.

40 Atkinson C, Stewart S, Upton PD, Machado R, Thomson JR, Trembath $\mathrm{RC}$, et al. Primary pulmonary hypertension is associated with reduced pulmonary vascular expression of type II bone morphogenetic protein receptor. Circulation 2002; 105: 1672-8.

41 Yang X, Long L, Southwood M, Rudarakanchana N, Upton PD, Jeffery TK, et al. Dysfunctional Smad signaling contributes to abnormal smooth muscle cell proliferation in familial pulmonary arterial hypertension. Circ Res 2005; 96: 1053-63.

42 Din S, Sarathchandra P, Yacoub MH, Chester AH. Interaction between bone morphogenetic proteins and endothelin-1 in human pulmonary artery smooth muscle. Vasc Pharmacol 2009; 51: 344-9. 